\title{
RD50 Collaboration - recent results on developing very high radiation resistant silicon detectors
}

\author{
Otilia Militaru $^{1^{*}}$ \\ Université catholique de Louvain \\ Louvain la Neuve, Belgium \\ E-mail: otilia.militaruluclouvain.be
}

\section{On behalf of the RD50 Collaboration}

For the luminosity upgrade of the Large Hadron Collider (SLHC), the present LHC experiment tracking systems, based on semiconductor detectors, need to be replaced. The new challenge will be the extreme radiation hardness requirement of up to $1 \times 10^{16} \mathrm{n}_{\mathrm{eq}} / \mathrm{cm}^{2}$.

The detectors will suffer from high radiation damage during their life time and will require radiation hard technologies to guarantee accurate track reconstruction. Current silicon sensors can operate up to $10^{15} \mathrm{n}_{\mathrm{eq}} / \mathrm{cm}^{2}$ because their radiation degradation could be balanced by increasing the oxygen amount of Float-Zone silicon substrates and high operating voltages are possible due to multi guard-rings geometry. Such devices are installed and running in the CERN Large Hadrons Collider (LHC) experiments. In the frame of the RD50-CERN Collaboration much work is in progress to extend this limit to $10^{16} \mathrm{n}_{\mathrm{eq}} / \mathrm{cm}^{2}$ in order to use silicon technology also in experiments at the proposed SLHC. This paper presents recent results of the RD50Collaboration on radiation hard technologies developed on Float Zone, epitaxial and Magnetic Czochralski n- and p-type silicon sensors. Their charge collection efficiency after proton, neutron and mixed irradiation has been determined and new technology 3D detectors have been produced and studied as well. For high fluences microscopic defects have been also investigated and compared to the detection capability of the irradiated sensors.

9th International Conference on Large Scale Applications and Radiation Hardness of Semiconductor Detectors-Rd09

Florence, Italy

30 September - 2 October 2009

Speaker

* A complete author list can be found at http://cern.ch/rd50/ 


\section{Introduction}

At the CERN Large Hadron Collider (LHC) the tracking detectors will be exposed to very high particles fluences for an integrated luminosity of $500 \mathrm{fb}^{-1}$ corresponding to 10 years of LHC operation. The detectors use silicon as base material for tracking systems and were designed to withstand a fluence of few $10^{15} \mathrm{n}_{\mathrm{eq}} / \mathrm{cm}^{2}$ for inner pixel layers, and few $10^{14} \mathrm{n}_{\mathrm{eq}} / \mathrm{cm}^{2}$ for outer strip layers. Much effort went into the design and in construction of such detectors and the research phase that preceded the construction gives us the guarantee that the sensors will stand the 10-years LHC running. The LHC upgrade proposal (SLHC), sustained by strong physics motivations, with the anticipated accumulated luminosity of $3000 \mathrm{fb}^{-1}$, imply a new R\&D program to find the best solution for the new very-hard radiation silicon detectors, that will have to stand an integrated fluence up to $10^{16} \mathrm{n}_{\mathrm{eq}} / \mathrm{cm}^{2}$. At small radii the damage is dominated by fast charged hadrons (mostly pions) while for $\mathrm{r} \geq 20 \mathrm{~cm}$ the neutrons originating from the calorimeter prevail [1].

The RD50 Collaboration "Development of Radiation Hard Semiconductor Devices for Very High Luminosity Colliders" is developing radiation resistant tracking sensors for the tracker upgrades at SLHC experiments. The challenges arise not only by the unprecedented high radiation level the detectors have to stand, but also from the high track density that need fast and high granularity detectors. The pixel layers will have to be extended up to higher radii from the interaction point, whereas the strip sensors will have to be changed, reducing in strip dimension, making less significant the distinction between pixels and strips as, e.g., the novel design "strixels" geometry.

Several approaches are already taken into account by the collaboration. The first one regards the "Defect and Material Characterization" that involve the characterization through different methods of the irradiation-induced different type defects in silicon material. It overlaps with the direction of "New materials" [2] that takes into account the possible use of other substrates than silicon, like silicon carbide and gallium nitride for improving the radiation hardness of detector. The next direction is the "Defect Engineering" that studies the possible ways to manipulate the defects induced by radiations. These ways could be by oxygenating the FZ (float zone) silicon, using $\mathrm{Cz}$ (Czochralski) and $\mathrm{MCz}$ (magnetic-Czochralski) silicon, EPI (epitaxial) silicon in order to optimize different parameters, like sensitivity of the detector (charge collection efficiency performance) and carriers lifetime and dynamic range. The other research lines regard the "Pad Detector Characterization", "New Structures" and "Full Detector Systems", respectively. In the following, few recent results will be described, with special regard on different options of detector material and technology for SLHC phase. This paper cannot describe the all recent activity of the collaboration. The extended presentation of all the research directions and the groups involved, together with a list of publications, can be found in [3], in recent Collaboration workshops [4, 5] and recent conferences [6, 7].

\section{Radiation effects in silicon}

The silicon is the base support for most of the solid-state tracking detectors of the experiments at LHC. The well understanding of the damage mechanisms produced by different type of particles in semiconductor is fundamental in obtaining effective detectors for such high doses. The damages can be divided in two main categories: surface damage that modifies the strips isolation and changes the interstrip capacitance affecting the readout noise and bulk damage that affects the silicon crystal and induces critical changes in detector electrical parameters. 


\subsection{Surface damage}

The surface damage is generated by ionizing energy loss (IEL) in the silicon oxide layer that covers the silicon. This damage that creates positive fixed charge in the oxide engenders a negative sign charge accumulation layer at the silicon surface, between the strips, that affects the total capacitance of the strips. Due to the significantly increase of the electric field around the strip junction, this damage might affect the breakdown voltage of the detector.

\subsection{Bulk damage}

Hadron radiation cause bulk crystal defects in a semiconductor due to Non-IonizingEnergy-Loss (NIEL). These defects have as consequence the increase of bulk leakage current and the decrease of the lifetime of free carriers proportional to the absorbed dose. Due to the creation of trapping centres in the band gap induced by the crystal defects, the charge carriers are trapped, leading to a gradually reduction of the effective drift length both for electrons and holes and therefore to a reduction of the charge collection efficiency of the detector. Another critical effect is the change of the effective doping concentration of the silicon bulk that implies both the modification of the full depletion bias voltage, and the space charge sign inversion, that bring other critical consequences. For leakage current and trapping effects no major distinction has been observed between different materials, whereas the effective doping concentration evolution varies strongly with different silicon material type. Each of these effects undergoes an annealing effect, if the irradiated material is kept at room temperature. The leakage current and electrons trapping modifies in a beneficial way but the holes trapping continue to increase with time $[8,9]$. The effective doping concentration will undergo "reverse annealing", meaning that the negative space charge is further increased. This is a prejudicial effect in standard FZ silicon but for $\mathrm{Cz}, \mathrm{MCz}$ or epitaxial silicon it can be beneficial [10].

\section{Silicon types and materials studied by RD50}

Different materials are presently investigated by RD50 Collaboration, like FZ-grown silicon, diffusion oxygenated FZ (DOFZ), Cz, and MCz silicon material, p-in-n, n-in-n and n-in$\mathrm{p}$ type substrates detectors. Another option is EPI-standard and EPI-diffusion oxygenated (EPIDO) silicon grown on $\mathrm{Cz}$ substrate, with different oxygen concentration and thicknesses (75, 100 and $150 \mu \mathrm{m})$. These are only few examples and all different combinations of these properties are also considered. The material mostly used for detector applications is the high resistivity FZ single crystal silicon. The n-type FZ, with p+ segmentation for strips, is the mostly used as tracking detector bulk material. It was seen that during irradiation the net space charge changes from positive to negative due to the radiation-induced creation of deep acceptors. After type-inversion, the junction is moved to the non-structured $n+$ uniform contact, thus an increasingly bias voltage is needed to fully deplete the silicon bulk. The increase of the space charge depends also on the silicon material. For high fluences a "double-junction" appears due to a non-homogeneous occupation of deep donors and acceptors in the space charge region leading to double peak electric field profile [11, 12]. The p-type bulk material has many advantages demonstrated by different groups $[13,14]$. The main advantage in using such substrates is the fact that the high electric field stay after irradiation at the structured side of the detector, as p-type material does not type-invert. In this case electrons are collected at the electrodes; having three times higher mobility than holes and therefore trapping is less effective it leads to a smaller degradation of the charge collection efficiency.

It is largely agreed [15] that a high concentration of oxygen in silicon has as consequence much higher radiation resistance for charged hadrons, gamma and electrons. FZ material, by default, has a very low concentration of oxygen $\left(<10^{16} \mathrm{at} / \mathrm{cm}^{3}\right)$ but there are several methods to increase the amount of oxygen by high-temperature diffusion of oxygen from $\mathrm{SiO}_{2}$ 
(DOFZ silicon, with oxygen concentration $2 \times 10^{17} \mathrm{~cm}^{-3}$ ) $[16,17]$. Nevertheless, the oxygen concentration is not homogenous inside the bulk for DOFZ as was presented in [13,14]. Cz and $\mathrm{MCz}$, owed to their specific growing method, have much higher oxygen content, $5-10 \times 10^{17} \mathrm{~cm}^{-3}$ than FZ and DOFZ silicon. Therefore, $\mathrm{Cz}$ and $\mathrm{MCz}$ silicon are extensively studied by the collaboration and the results of irradiations with different particles (protons from $10 \mathrm{MeV}$ up to $23 \mathrm{GeV}[18,19,20]$, reactor neutrons [21], $900 \mathrm{MeV}$ electrons [22], and gammas [21]) show a clear superiority of this material in terms of radiation hardness with respect to conventional FZ and DOFZ. After neutron irradiation, the stable damage component of the effective doping concentration is usually of negative sign independent of the silicon type. The only exception observed so far is n-type epitaxial detectors $(50,75 \mu \mathrm{m})$ of low resistivity [23] where no space charge sign inversion is observed and effective donors are generated when irradiated with neutrons. In Fig.1 $[23,24]$ can be seen the variation of the $\mathrm{N}_{\text {eff }}$ (effective doping concentration) on $300 \mu \mathrm{m}$ thick wafers for different materials and suppliers, with the increased neutron fluence. On the same material, MCz-p,n detectors processed by Micron show smaller increase in $\mathrm{V}_{\mathrm{fd}}$ (the bias voltage at which the detector is fully depleted of mobile carriers) than other suppliers meaning that the introduction rate of stable damage after type inversion for Micron, for example, is smaller. This effect can be caused by the processing particularities of each company, but these results need further investigation.

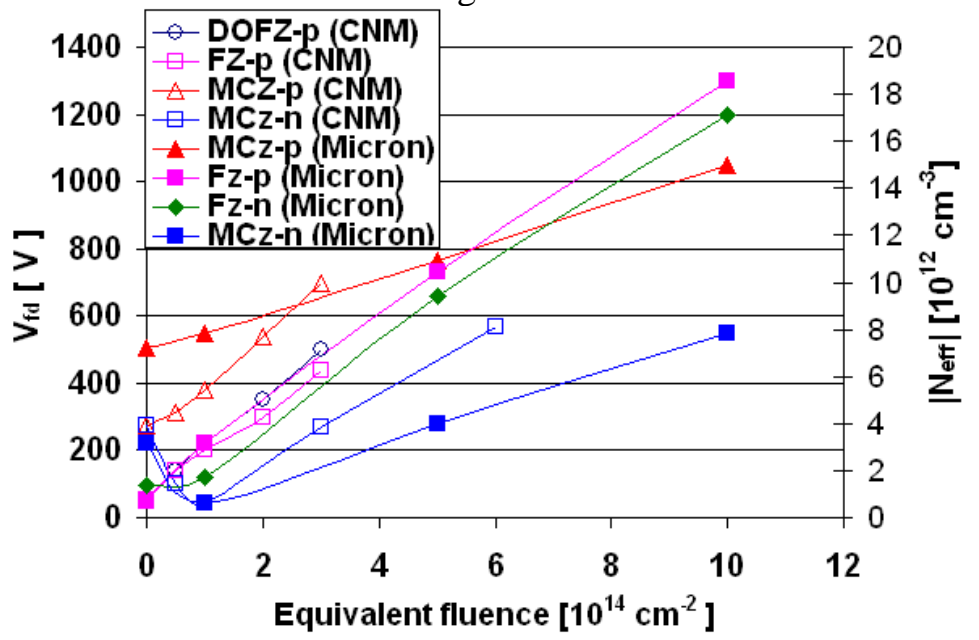

Fig 1. Dependence of $V_{f d}$ on fluence for different detectors processed by several manufacturers

For very high fluences $\left(>10^{15} \mathrm{n}_{\mathrm{eq}} / \mathrm{cm}^{2}\right)$ the charge collection is strongly limited by the trapping effects. Therefore detectors with short drift lengths, to minimize the distance on which the mobile charge carriers are collected, are intensively studied by collaboration. In 3D geometry the detector thickness is decoupled from the distance for charge collection, having the electrodes on column shape. In some geometry the columns get deep into the silicon bulk from one side 3D-STC (Single Type Column) from front to back side or from both sides 3D-DDTC (Double Sided Double Type Column). For other geometry the columns traverse entirely the silicon bulk. Both CNM Barcelona and FBK Trento have successfully produced these kind of detectors on n-type and p-type substrate and are now under tests [25, 26].

\section{Charge collection efficiency on very high irradiated devices}

In RD50 Collaboration intensive studies were carried out concerning the evaluation of charge collection efficiency (CCE) on different materials after irradiation with several types of particles. Recent measurements on strip sensors with $n+$ readout and p-type substrate at superLHC fluences $[27,28]$ were reported. Very high collected charge was observed after irradiation, even with detectors irradiated by $1 \times 10^{16} \mathrm{n}_{\mathrm{eq}} / \mathrm{cm}^{2}$, as shown in Fig 2 . 

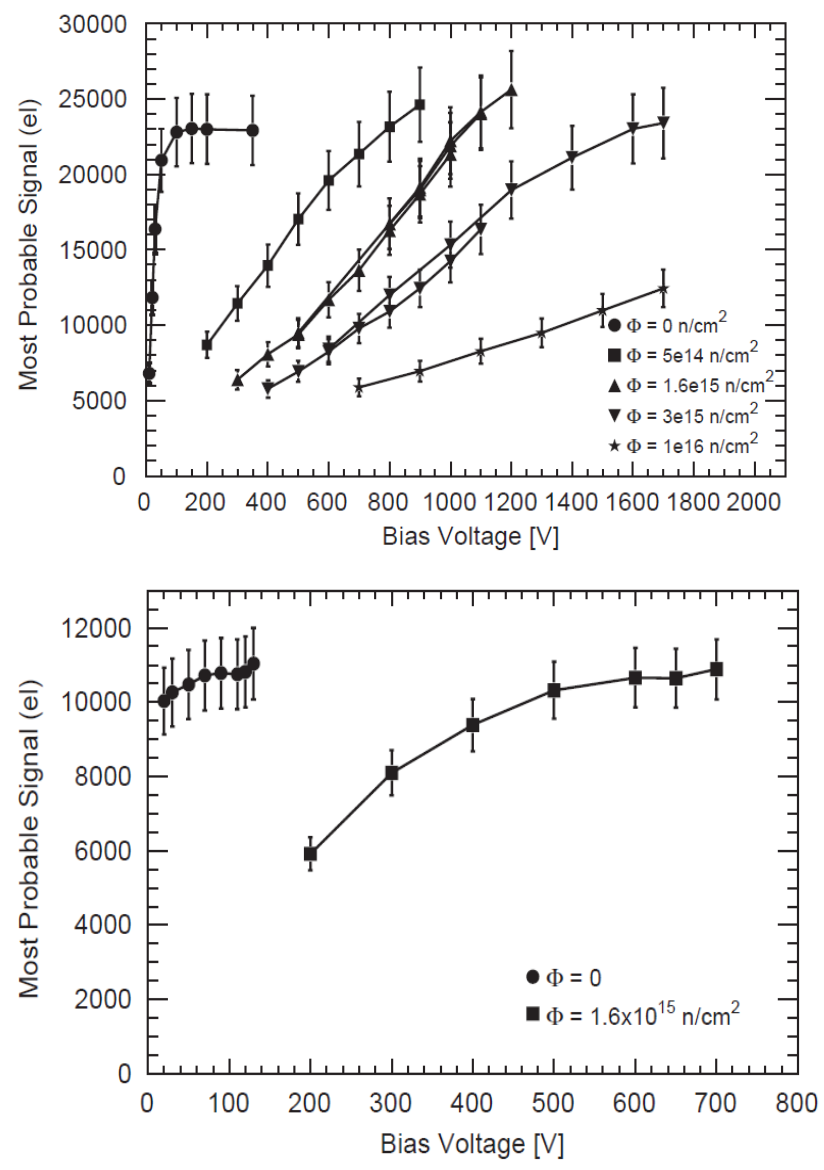

Fig.2 Most probable signal value as a function of bias voltage for irradiated 300 (upper) and 140 (lower) $\mu \mathrm{m}$-thick detectors, at $-20^{\circ} \mathrm{C}$ for different fluences [27].

Simulations were done by Ljubljana group [27], calculating the charge generation by a MIP in similar structure considering the effective trapping time $\mathrm{t}_{\text {eff }}$ for $\mathrm{e}$ - and holes, $1 / \mathrm{t}_{\mathrm{eff}}\left(\mathrm{e}^{-}\right.$and $\left.\mathrm{h}\right)=\beta_{\mathrm{e}, \mathrm{h}}$ $\mathrm{x} \Phi\left(\Phi\right.$, particle fluence, $\left.\beta_{\mathrm{e}}=4.8 \times 10^{-16} \mathrm{~cm}^{2} / \mathrm{ns}, \beta_{\mathrm{h}}=5.3 \times 10^{-16} \mathrm{~cm}^{2} / \mathrm{ns}\right)$ and the results underestimate the experimental values. One of the possible explanation is the onset of the avalanche effects in sensors, which is triggered by an electric field higher than $10 \mathrm{~V} / \mu \mathrm{m}$ for electrons. It must be considered that in strip detectors this condition is satisfied sooner than in diodes, which might explain why this effect was not previously observed. Nevertheless, recent reports from other groups on 150 and $75 \mu \mathrm{m} \mathrm{Cz}$ epitaxial detectors [29] and on $300 \mu \mathrm{m}$ n-in-p Micron sensors [28, 31] show the same effect. In Fig. 3 [30] for $150 \mu \mathrm{m}$ EPI silicon can be seen a saturation of CCE for low fluences whereas for high fluences a strong increase can be observed if high bias voltage is applied. It was explained by a multiplication of charge if the electric field is high enough inside the detector. This result is confirmed on $75 \mu \mathrm{m}$ EPI silicon detectors where the electric field is higher for the same bias voltage with respect to thicker substrates. Using alpha particles or laser with different penetration length can be seen different degree of charge multiplication, the smaller the penetration depth is, the higher the charge multiplication. Therefore the multiplication zone can be localised at the front side of the structure as expected because there is the p-n-junction with the high electric field. Investigation of these effects will be one of the major objectives of the collaboration for the following years. 


\section{Mixed irradiations (charged hadrons and neutrons)}

Much effort was devoted lately also to understand the damage after irradiation of detectors exposed to mixed irradiation fields resembling those of LHC experiments. Combined irradiations were performed by fast charged hadrons (pions or protons), followed by additional neutron irradiations $[32,33,34,35]$. It was seen that in case of $\mathrm{n}-\mathrm{MCz}$ material, a negative space charge is introduced by neutrons, whereas positive space charge is introduced by protons irradiations. Therefore combined irradiations introduce defects that compensate.
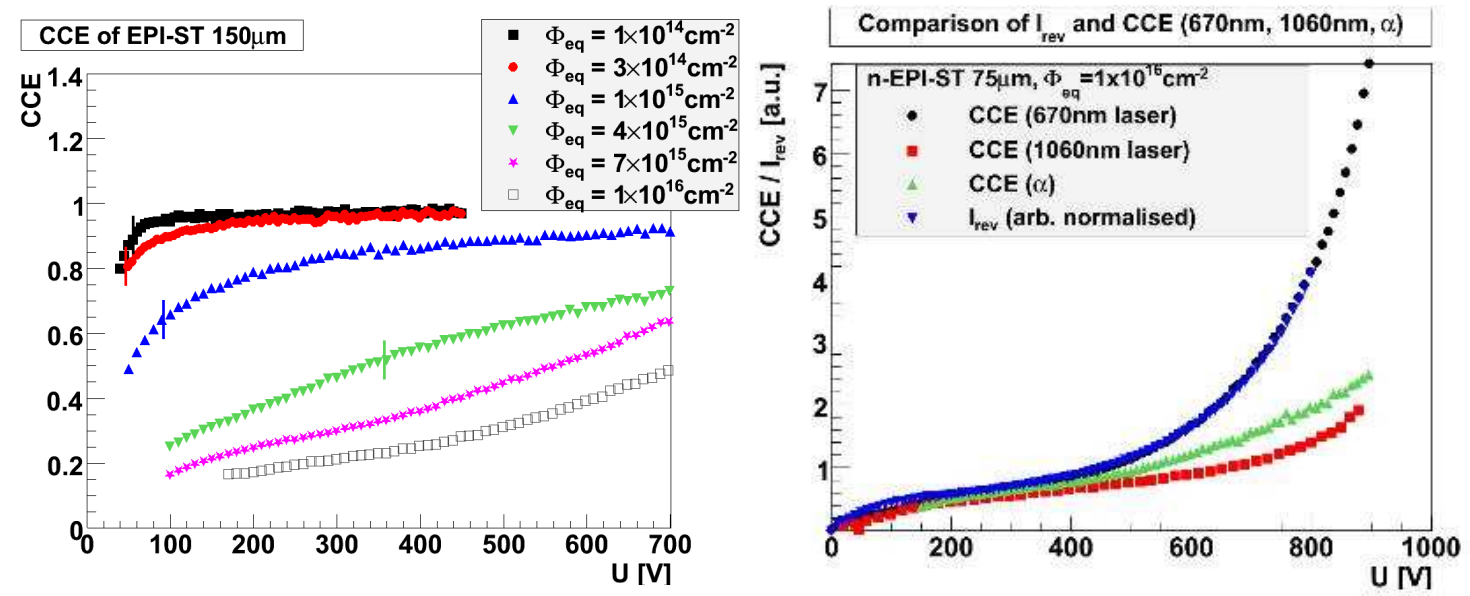

Fig.3 The charge collection efficiency measured (varying the bias voltage $U(V)$ ) with alpha particles on EPI $150 \mu \mathrm{m}$ (left) and alpha particle and laser on EPI $75 \mu \mathrm{m}$ (right) sensors (compared with a reversed leakage current), after complete beneficial annealing [30]

This issue must be exploited when considering the layers at distances from the interaction point of the experiments that withstand equal proton and neutron fluxes. In such case, the defects introduced could cancel the increase of the negative space charge. In Fig. 4 [32] n-type FZ and $\mathrm{MCz}$ silicon sensors were irradiated with nuclear reactor neutron and with $23 \mathrm{GeV}$ protons. Both FZ and MCz show the predicted behavior with mixed irradiations, the FZ doses effects are cumulative as $\mathrm{N}_{\text {eff }}$ increases, whereas for $\mathrm{MCz}$ the effects compensate as the effective doping concentration is diminished.

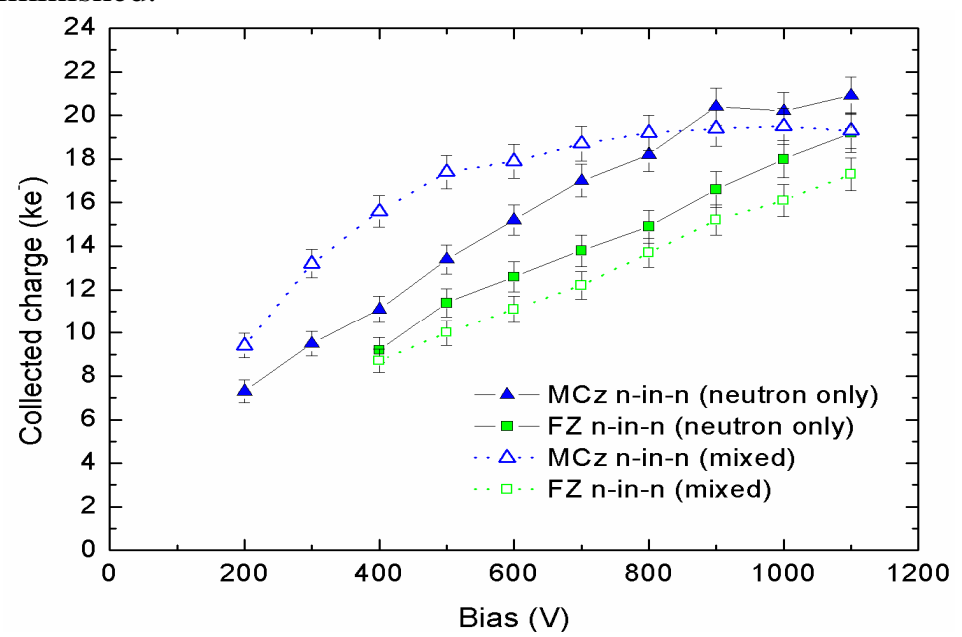

Fig 4. Charge collection measurements after mixed and neutron only irradiations of $M C z$ and FZ sensors [32]. 


\section{Conclusions}

The main goal of the RD50 Collaboration is to give an answer to the increased necessity to find suitable tracking silicon sensors acting in high radiation environment. A very wide variety of materials and types are tested in different particle environments and mixed irradiations and many testing systems were developed. Investigations done on p-type FZ material of charge collection efficiency up to fluences of $1 \times 10^{16} \mathrm{n}_{\mathrm{eq}} / \mathrm{cm}^{2}$ show very promising results and could be used for trackers upgrade due to their capacity of not type inverting. The results obtained by different groups showed that the radiation induced increase of effective space charge is different for $\mathrm{n}$-type $\mathrm{MCz}$ and EPI silicon as compared to FZ. In mixed neutron and proton irradiations, space charge compensation effects are evident. These results need further investigation for fully understand the prospective of this material as a possible candidate for SLHC experiments. A good choice would be for the radii in the experiments where the radiation environment is equally shared by both charged hadrons and neutrons and a possible partial cancellation effect of the degradation of $\mathrm{N}_{\text {eff }}$ is possible. Also measurements strongly suggest that high bias voltages could lead to a significantly improved charge collection performance of strip detectors at SLHC fluences. Many other tests are still ongoing with the aim of finding the optimum solution for the SLHC upgrade.

\section{References}

[1] http://cmsdoc.cern.ch/cms/archives/07/LHCC/slhc-eoi-final.pdf

[2] P.J. Sellin, J. Vaitkus, on behalf of RD50, Nucl. Instr. and Meth. A 557 (2006) 479-489

[3] RD50 Collaboration, RD50 Status Report 2008, to be published at CERN

[4] http://rd50.web.cern.ch/rd50/14th-workshop/

[5] http://indico.cern.ch/conferenceDisplay.py?confId=65918

[6] http://villaolmo.mib.infn.it/Information_2009.html

[7] http://www.nss-mic.org/2009/NSSMain.asp

[8] G. Kramberger et al., Nucl. Instr. and Meth. A 481 (2002) 297

[9] O. Krasel, Proceedings of the first RD50 Workshop, Oct 2002 (see RD50, http://www.cern.ch/rd50)

[10] M. Moll, Nucl. Instr. and Meth, A 565 (2006) 202

[11] M. Swartz et al., "Observation, modelling and temperature dependence of doubly peaked electric fields in irradiated silicon pixel sensors", Nucl. Instr. and Meth. A proceedings doi:10.1016/j.nima.2006.05.002

[12] V.Eremin, E.Verbitskaya, Z.Li, Nucl. Instr. and Meth. A476 (2002) 556

[13] A. Barcz, SIMS laboratory, Physics Institute of the Polish Academy of Science, Warsaw, Poland

[14] G. Lindström, I. Dolenc, E. Fretwurst, F. Hönniger, G. Kramberger et al., Nucl. Instr. and Meth. A 568 (2006) 66-71

[15] 3rd RD48 status report. CERN-LHCC-2000-009

[16] G.Casse, et al., Nucl.Instr.and Meth., A 438 (1999) 429 
[17] G.Lindstrom et al., Nucl. Instr.and Meth. A 466 (2001) 308

[18] G. Pellegrini, M. Ullán, J.M. Rafi, C. Fleta, F. Campabadal, M. Lozano, Nucl. Instr. and Meth. A $552(2005) 27$

[19] J. Härkönen et al., Nucl. Instr and Meth, A 518 (2004) 346

[20] A.G.Bates, M.Moll, Nucl. Instr. and Meth, A555 (2005) 113

[21] Z. Li, M. Bruzzi, V. Eremin, J. Harkonen, J. Kierstead, P. Luukka, D. Menichelli, E. Tuominen, E. Tuovinen, E. Verbitskaya, Nucl. Instr. And Meth, A 552 (2005) 34

[22] S. Dittongo, L. Bosisio, D. Contarado, G. D’Auria, E. Fretwurst, J. Härkönen, G. Lindström, E. Tuovinen, Nucl. Instr. and Meth. A 546 (2005) 300

[23] E. Fretwurst et al.,"Comparison of neutron damage in thin FZ, MCz and epitaxial silicon detectors", presented at 10th RD50 Workshop, Vilnius, June, (2007)

[24] G. Kramberger et al.,"Charge collection measurements on MICRON RD50 detectors", ATLAS ID upgrade, Workshop, Valencia, (2007)

[25] A.Zoboli et al, Nucl. Instr. And Meth A604 (2009) 238-241

[26] C.Fleta et al., Nucl. Instr. And Meth A607 (2009) 89-91

[27] I.Mandic et al. Measurement of anomalously high charge collection efficiency in $n+p$ strip detectors irradiated by up to $10^{16}$ neq/cm2 NIM A 603 (2009) 263-267

[28] G. L. Casse et al.,"Radiation Hardness of p-type detectors", Presented at 7th RESMDD Conference, Florence, 2008.

[29] J.Lange at al. $14^{\text {th }}$ RD50 workshop Freiburg, 2009

[30] J. Lange et al., "Charge collection studies of proton-irradiated n- and p-type epitaxial silicon detectors", 11th European Symposium on Semiconductor Detectors, Wildbad Kreuth, Germany, June 2009

[31] G. Casse at al., 14th workshop of RD50 Collaboration, Freiburg 5-7 June 2009.

[32] T. Affolder at al., " Charge Collection, Power, and Annealing Behaviour of Planar Silicon Detectors after Reactor Neutron, Pion and Proton Doses up to $1.6 \times 10^{16}$ neq $\mathrm{cm}-2$ " presented at 13th RD50 Workshop, CERN, November, 2008.

[33] G. Krambeger et al.,"C-V/I-V and CCE measurements of MCz and FZ p and n type diodes after mixed irradiations", presented at 12th RD50 Workshop, Ljubljana, June, 2008

[34] R. Eber et al.,'TCT-Measurements of mixed irradiated Magentic Czochralski Diodes in the SLHCScenario", presented at 14th RD50 Workshop, Freiburg, June, 2009.

[35] T. Affolder at al., "Comparison of the CCE properties of microstrip detectors made with different substrates and irradiated with protons and neutrons at different temperature", presented at 14th RD50 Workshop, Freiburg, June, 2009. 